R. Massaro ${ }^{1}$, P. Roozemond ${ }^{2}$, M. D'Haese ${ }^{2}$, P. Van Puyvelde ${ }^{1}$ *

${ }^{1}$ Department of Chemical Engineering, KU Leuven, Leuven, Belgium

${ }^{2}$ DSM Materials Science Center, Geleen, The Netherlands

\title{
Flow-Induced Crystallization of Polyamide-6
}

\begin{abstract}
Flow-induced crystallization has been widely studied for a variety of polymers with the main focus on polyolefins. In this work, research has been conducted on the flow-induced crystallization of another important class of polymers, namely polyamides. Different polyamides-6 with varying molecular weight have been studied. At relatively modest values of shear rate, rheology has been used to study the kinetics of flow-induced crystallization. Typical scaling relations based on the longest relaxation time and the Rouse time - usually obtained for polyolefins - are tested for the polyamides under investigation in order to identify the different regimes of flow-induced crystallization. At high shear rates, a correct rheological signal was impossible to collect. However, the rheometer was used in this case to prepare the sample to be studied exsitu by Wide Angle X-ray Scattering experiments to determine the onset shear rate for the formation of highly oriented shishkebab structures.
\end{abstract}

\section{Introduction}

Polymers are very interesting materials from an engineering point of view since they are not costly, can display a wide variety of properties, and can be easily processed using a broad range of processing techniques. However, during this processing step, polymers are often subjected to a complex thermomechanical history that will determine the structure and hence the final properties of the polymeric part. This resulting structure is based on an interplay between the different time-scales involved in the process: (i) the intrinsic material time-scale as determined by the relaxation time spectrum of the polymer, (ii) the flow intensity expressed by the flow rate (i.e. either shear or extensional flow) and flow time and (iii) finally the thermal time scale as set by the cooling rate. The structure will hence be determined to a large extent by the orientation of polymeric chains imposed by the flow field and the crystallization kinetics under flow.

Nowadays, it is widely accepted that the crystallization kinetics can drastically be enhanced by applying flow, a phenomenon known as flow-induced crystallization (FIC) (e.g. Acierno and Grizzuti, 2008). In quiescent conditions, polymer crystallization typically occurs in a two step process: during a

\footnotetext{
* Mail address: Peter Van Puyvelde, Department of Chemical Engineering, KU Leuven, Celestijnenlaan 200F box 2424 Leuven, Belgium

E-mail: peter.vanpuyvelde@kuleuven.be
}

first step, small ordered volumes called nuclei are formed that - in a second step - will grow radially to form spherulites. The application of flow will basically affect the first step as it is known to increase the nucleation density. When the applied flow rate is sufficiently severe, even the morphology of the crystallites can be affected and instead of the classical spherulites, shish-kebab structures might form (Acierno and Grizzuti, 2008; Housmans et al., 2009; Lamberti, 2014; Van Meerveld et al., 2004).

Physically FIC can be explained by an increase in the polymer chain orientation and resulting liquid-solid free energy difference, which facilitates an enhanced nucleation rate (Acierno and Grizzuti, 2008; Acierno et al., 2008). Within this framework, typically three different flow regimes can be detected. The transition of regime is indicated by critical values of Deborah number, $\mathrm{De}=\tau / \mathrm{t}_{\exp }$, or Weissenberg number, $\mathrm{Wi}=\tau \dot{\gamma}$, defined with respect to molecular orientation (using $\tau_{\text {rep }}$ ) and molecular stretch (using $\tau_{\text {Rouse }}$ ) (Acierno and Grizzuti, 2008; Acierno et al., 2008; Housmans et al., 2009; Van Meerveld et al., 2004). Once those transition parameters are determined experimentally, they can be used in simulations for further improvement of processing conditions and prediction of the final product properties (Hamad et al., 2015; Ma et al., 2013).

Although the effect of shear deformation on the crystallization kinetics and structure development has gained a lot of interest over the last decades, researches have mainly been focusing on the behavior of polyolefins (Baert and Van Puyvelde, 2006; Baert et al., 2006; Kumaraswamy et al., 1999; Kumaraswamy et al., 2000; Liedauer et al., 1993; Somani et al., 2000; Seki et al., 2002; Vega et al., 2009; Vleeshouwers and Meijer, 1996;). The influence of different material properties (e. g. molecular weight) and flow related parameters were investigated (Acierno et al., 2003), proving that flow has a large influence on the crystallization of polyolefins as was already indicated above. Polyolefins have been ideal "model" polymers in this respect since additional factors such as moisture sensitivity or hydrogen bonding are typically absent in these materials. In other engineering polymer families, like for instance polyamides, the latter factors do become important. However, flowinduced crystallization of such polymers has not been largely studied, although some literature is available for both aliphatic and semi-aromatic polyamides, ester-amide block copolymer and nanocomposites (Acierno and Van Puyvelde, 2005; Gao et al., 2015; Kelnar et al., 2007; Naudy et al., 2007; Naudy and Fulchiron, 2007; Tavernier et al., 2008). Such polyamides are condensation polymers with typically a lower molecular weight with hence a less pronounced effect of flow on the crys- 
tallization. Also moisture plays a significant role in these polymers and drying conditions have proven to be crucial in this respect (Ebewele, 2000; Gowariker et al., 1986; Harper and Petrie, 2003; Kohan, 1973; Mark and Stafford Whitby, 1940). The drying conditions are critical as severe drying can also induce post-polycondensation reactions in the solid state that increase molecular weight and viscosity (Khanna et al., 1996).

In this paper, we focus on the flow-induced crystallization of polyamides with different molecular weight. The main target is to investigate whether the different regimes based on the Weissenberg number with respect to chain orientation and chain stretch can also be identified for the materials under investigation here. First classical rheological experiments will be performed as was also done before for polyolefins. However, rheology is limited to rather modest shear rates, at least if the rheological signal is used to follow the flow-induced crystallization kinetics. Because of the smaller molecular weight of the polyamides under study, the shear rates that need to be reached in order to stretch the chain are rather high and at those rates, no reliable rheological signal can be captured anymore. However, the rheometer could still be used to prepare the sample for further off-line analysis in, for instance, a Wide Angle $\mathrm{X}$-ray scattering setup.

\section{Materials and Methods}

Three commercial grades of polyamide-6 (PA-6) were provided by DSM Materials Science Center (Geleen, The Netherlands) as model polymers for the study. The polymers contain a small amount of a mould release agent to make the recuperation of the sample possible after rheological measurements. The samples do not contain any significant amounts of nucleating agents. The melting temperature of the various samples was determined by DSC and was found to be around $220^{\circ} \mathrm{C}$. The molecular weights of the samples under investigation are shown in Table 1.

The polymers, available in pellets, were pressed using a Collin plate press, to create disks with a diameter of $25 \mathrm{~mm}$. Before pressing, the pellets were subjected to a drying protocol as explained further. During pressing, pellets were molten at $240^{\circ} \mathrm{C}$ under vacuum for $3 \mathrm{~min}$ in order to ensure complete melting of the crystalline phase. Then the pressure was increased to 70 bar and after 4 min cooled to a temperature of $40^{\circ} \mathrm{C}$ to remain below the PA- 6 glass transition temperature. Before performing any measurement, the samples were dried in a vacuum oven at $80^{\circ} \mathrm{C}$ for $24 \mathrm{~h}$ in order to remove possible water traces (same drying protocol was used for the pellets). The temperature should not exceed $110^{\circ} \mathrm{C}$ to avoid polycon-

\begin{tabular}{|c|c|c|}
\hline Polymer & $\begin{array}{c}\mathrm{M}_{\mathrm{n}} \\
\mathrm{kg} / \mathrm{mol}\end{array}$ & $\begin{array}{c}\mathrm{M}_{\mathrm{w}} \\
\mathrm{kg} / \mathrm{mol}\end{array}$ \\
\hline PA1 & 16.5 & 33 \\
PA2 & 28.5 & 57 \\
PA3 & 33 & 66 \\
\hline
\end{tabular}

Table 1. Molecular weight characteristics of the polymers used in this work densation, since this would change the molecular weight distribution of the polymer (Khanna et al., 1996). When the dried samples were taken out of the oven, they were stored in a desiccator with silica gel to prevent a further uptake of water.

Rheological characterization was performed in the linear viscoelastic regime using a strain-controlled rotational rheometer (ARES-Melts, TA Instruments), with a parallel plate geometry (plate diameter $25 \mathrm{~mm}$, gap thickness $1 \mathrm{~mm}$ ). All measurements were carried out in a nitrogen atmosphere to prevent moisture absorption and thermal degradation of the sample. Moduli were measured with the dynamic frequency scanning from $0.1 \mathrm{rad} / \mathrm{s}$ to $100 \mathrm{rad} / \mathrm{s}$ at $5 \%$ strain.

To perform the quiescent crystallization and flow-induced crystallization experiments, the same rheometer was used but now equipped with a cone and plate geometry (plate diameter $25 \mathrm{~mm}$, cone angle $0.1 \mathrm{rad}$, truncation $52 \mu \mathrm{m}$ ) to obtain a well-defined shear rate. In each experiment, the short-term shearing temperature protocol as pioneered by JaneschitzKriegl et al. (1993) (shown in Fig. 1) was followed. The sample was loaded and kept for $15 \mathrm{~min}$ at an annealing temperature of $240{ }^{\circ} \mathrm{C}$ (substantially above the nominal melting point of $220^{\circ} \mathrm{C}$ ) to erase the thermo-mechanical history of the polymer. The annealing temperature has to be high enough to guarantee the complete melting of the sample, but must not be too high to avoid its degradation. After this time, the sample was cooled down with a rate of $5^{\circ} \mathrm{C} / \mathrm{min}$ until the crystallization temperature was reached. This is a trade-off between measurable kinetics and a reasonable crystallization time. Zero time scale was assigned to the instant at which the crystallization temperature was reached. Three different temperatures of crystallization of $198^{\circ} \mathrm{C}, 200^{\circ} \mathrm{C}$ and $202^{\circ} \mathrm{C}$ were used although the majority of the results shown in this paper were collected at $202^{\circ} \mathrm{C}$.

For quiescent crystallization experiments, the three crystallization temperatures are the relevant parameters. However, when studying flow-induced crystallization, also the shear rate and shear time need to be specified. Here, once the crystalliza-

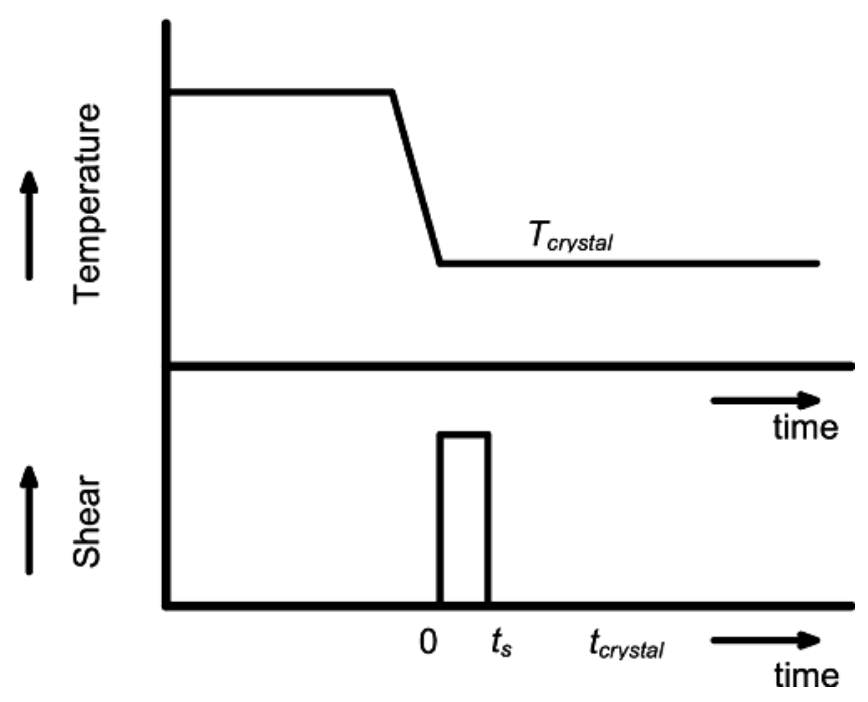

Fig. 1. Temperature and shearing protocol used to study flow-induced crystallization

Intern. Polymer Processing XXXIII (2018) 3 
tion temperature was reached, a constant shear rate, $\dot{\gamma}$, was applied for a shearing time, $\mathrm{t}_{\mathrm{s}}$ of $1 \mathrm{~s}$. To follow the crystallization, two types of experiments were conducted. At small values of the shear rate, where the rheological signal could still be interpreted, the modulus of the complex viscosity, $\eta^{*}$, was followed as a function of time in order to determine a characteristic time for crystallization. However, at too high values of the shear rate, the rheological signal is useless because of shear fracture and cannot be used anymore to follow the crystallization kinetics. In this case, in order to identify possibly oriented structures, the rheometer was used to impose a well-defined shear rate. After shearing, the temperature was immediately decreased till room temperature to recuperate the oriented samples. The latter was then analyzed off line by means of Wide Angle X-ray Scattering (WAXS).

The two dimensional (2D) WAXS experiments were carried out on a Ganesha 300XL+ system from JJ X-ray, Copenhagen, Denmark. The instrument is equipped with a detector (model Pilatus 300 K, Dectris, Baden-Daettwil, Switzerland), with a pixel size of $172 \mu \mathrm{m} \times 172 \mu \mathrm{m}$. The X-ray source is a sealed tube X-Ray Cu-source (model Genix 3D Microfocus, Xenocs, Sassenage, France) with integrated monochromator (multilayer optic "3D version" optimized for SAXS) $(30 \mathrm{~W})$. The wavelength used is $\lambda=1.5408 \AA$. The detector moves within a vacuum chamber with sample-to-detector distance varied between $0.115 \mathrm{~m}$ and $1.47 \mathrm{~m}$ depending on the configuration used, as calibrated using silver behenate $\left(\mathrm{d}_{001}=58.380 \AA\right)$. The minimized background scattering, a small beam stop and a high performance detector allows for a detectable q-range varying from $3 \times 10^{-3}$ to $3 \AA^{-1}(0.2$ to $210 \mathrm{~nm})$. The scattering patterns were collected with sample to detector distance of $120 \mathrm{~mm}$.

\section{Results and Discussion}

\subsection{Basic Rheological Characterization and Determination of Transition Times}

A linear viscoelastic characterization of PA-6 was performed in a narrow temperature range of 225 to $250{ }^{\circ} \mathrm{C}$. The latter was chosen based on the limited thermal stability of polyamides at temperatures significantly higher than their $T_{m}$ and on the fast crystallization rates at temperatures lower than their $\mathrm{T}_{\mathrm{m}}$ (Acierno and Van Puyvelde, 2005). Starting from the dynamic moduli at those temperatures, master curves for the three grades were obtained, at a reference temperature of $240^{\circ} \mathrm{C}$ using the timetemperature superposition principle (TTS). Only the horizontal shift procedure was adopted which is justified based on the relatively small temperature window. The relation between the horizontal shift factor, $\alpha_{\mathrm{T}}$, and the temperature $\mathrm{T}$ is given by the Arrhenius expression (Ferry, 1980):

$\log \alpha_{\mathrm{T}}=\frac{\mathrm{E}}{\mathrm{R}}\left(\frac{1}{\mathrm{~T}}-\frac{1}{\mathrm{~T}_{\text {ref }}}\right)$,

with $T_{\text {ref }}$ the reference temperature to which we choose to reduce the experimental data, $\mathrm{E}$ the activation energy and $\mathrm{R}$ gas constant. The value of activation energy of $55 \mathrm{~kJ} / \mathrm{mol}$ was provided by DSM. Hence, a simple Arrhenius expression is suffi- cient to obtain the shift factor, $\alpha_{\mathrm{T}}$. As the activation energy value seems to be quite constant with molecular weight, it was decided to use the same value of the constants for the three grades. The resulting master curves are shown in Fig. 2.

From the master curve of the storage and loss moduli, it is possible to define the longest relaxation time, $\tau_{\text {rep. }}$ At low frequencies in the terminal region, the storage modulus $G$ ' has a limiting slope of two whereas the loss modulus G" obtains a slope of one. The inverse of the frequency value at which the slopes deviate from these values is a good indication of the longest relaxation time, $\tau_{\text {rep, }}$ at the reference temperature, $\mathrm{T}_{\text {ref }}$ (Ferry, 1980). Moreover, it should be said that this method is not very accurate in determining the reptation times, since it is generally admitted that the latter vary with the power 3.4 of the molecular weight. This is, however, a very practical approach to evaluate the time scale of the crystallization process. When deforming the polymer slower than $1 /$ longest relaxation time, the material has time to relax and behaves like a Newtonian fluid. However, if the material is deformed faster than 1/longest relaxation time, orientational effects will start to play a role, which - in this study - might affect the crystallization kinetics. Hence, this value of $\tau_{\text {rep, }}$ will be used to determine the first characteristic Weissenberg number, $\mathrm{Wi}_{\text {rep }}$, that will yield the transition between the shear rate ranges that do not influence the crystallization kinetics and the shear rate ranges at which orientation effects will start to dominate the crystallization.

Based on the master curves another important characteristic time, the Rouse relaxation time, $\tau_{\text {Rouse }}$, can be obtained. This latter relaxation time will become important in the present work, in order to determine the transition to stretched chains which could lead to the formation of highly oriented shish-kebab structures through the Weissenberg number, $\mathrm{Wi}_{\mathrm{s}}$. This Rouse relaxation time, $\tau_{\text {Rouse }}$, can be determined once the longest relaxation time, $\tau_{\text {rep }}$, is known using the following expression:

$\tau_{\text {rep }}=3 Z \tau_{\text {Rouse }}=3 \frac{M_{w}}{M_{\text {entanglement }}} \tau_{\text {Rouse }}$,

with $\mathrm{Z}=\left(\frac{\mathrm{M}_{\mathrm{w}}}{\mathrm{M}_{\text {entanglement }}}\right)$ the number of entanglements per chain, $\mathbf{M}_{\mathrm{w}}$ the average molecular weight of the polymer and $\mathrm{M}_{\text {entanglement }}$ the molecular weight between the entanglements (Baert et al., 2006; Housmans et al., 2009; Van Meerveld et al., 2004). The entanglement molecular weight used for PA-6 was $2.470 \mathrm{~kg} / \mathrm{mol}$. As was explained above, these relaxation times were determined at the reference temperature of $240{ }^{\circ} \mathrm{C}$. To determine these transition times at different temperatures, the time-temperature superposition factor $\alpha_{T}$ can be used (Ferry, 1980).

The full characterization of all transition times at the different temperatures examined in this work and of the different polymers can be found in Appendix A. Table 2 summarizes the transition times at a temperature of $202{ }^{\circ} \mathrm{C}$ which was used as the crystallization temperature in the experiments under flow. From this table, the dependence of $\tau_{\text {rep }}$ on temperature and molecular weight is clear. As expected $\tau_{\text {rep }}$ decreases as molecular weight $\mathrm{M}_{\mathrm{w}}$ decreases since shorter chains relax faster than longer chains. On the other hand, $\tau_{\text {rep }}$ increases when decreasing the temperature $\mathrm{T}$ (see appendix $\mathrm{A}$ ) because of the reduced mobility of the chains. 


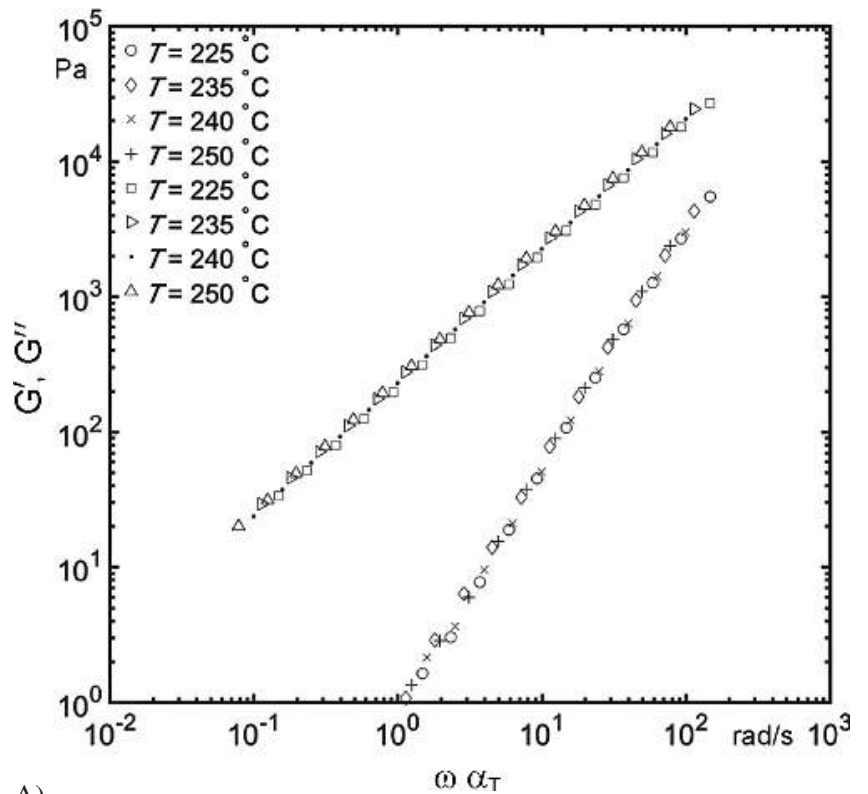

A)

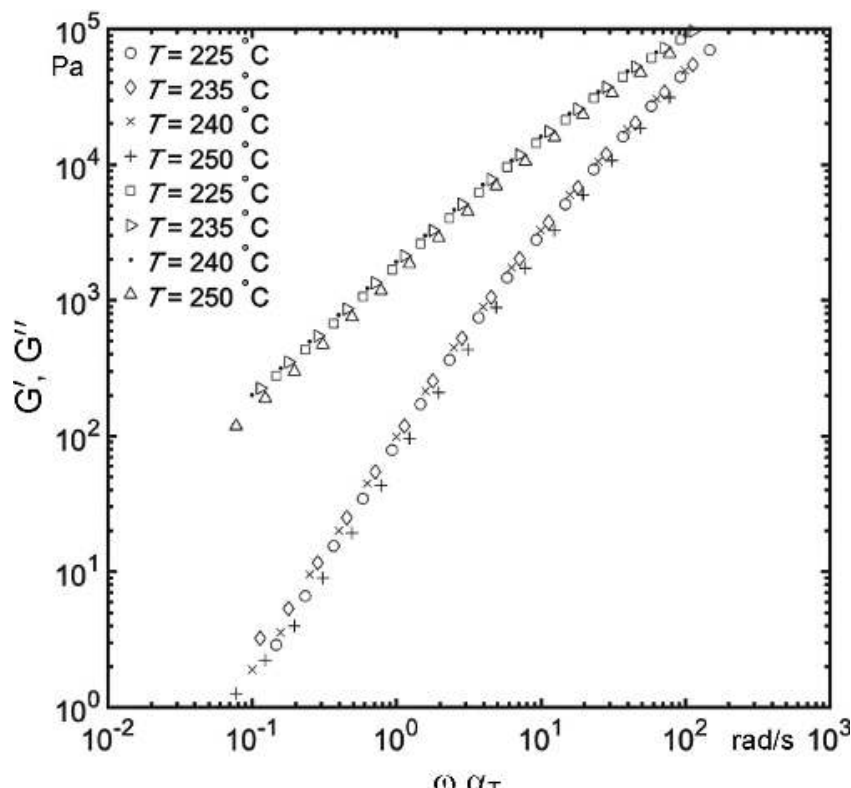

C)

Fig. 2. Storage and loss moduli master curve at a reference temperature of $240^{\circ} \mathrm{C}$ of $A$ ) $\left.\left.P A 1, B\right) P A 2, C\right) P A 3$

\begin{tabular}{|c|c|c|c|}
\hline Polymer & $\begin{array}{c}\mathrm{M}_{\mathrm{w}} \\
\mathrm{kg} / \mathrm{mole}\end{array}$ & $\begin{array}{c}\tau_{\text {rep }} \\
\mathrm{s}\end{array}$ & $\begin{array}{c}\tau_{\text {Rouse }} \\
\mathrm{s}\end{array}$ \\
\hline PA1 & 33 & 0.073 & $1.83 \cdot 10^{-3}$ \\
PA2 & 57 & 0.183 & $2.65 \cdot 10^{-3}$ \\
PA3 & 66 & 0.393 & $4.90 \cdot 10^{-3}$ \\
\hline
\end{tabular}

Table 2. Rheological time-scales at $T=202^{\circ} \mathrm{C}$

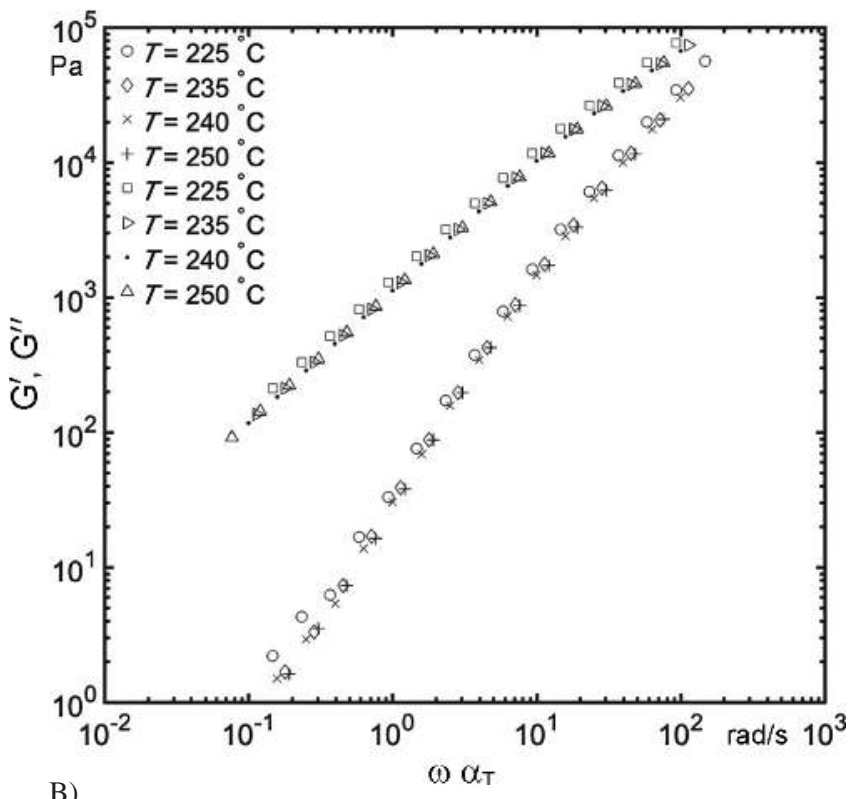

\subsection{Quiescent Crystallization}

Although quiescent crystallization kinetics, as well as its dependency on molecular weight and temperature, are rather well understood, the knowledge of its time-scale remains necessary in order to compare with later flow-induced crystallization results. The global kinetics of quiescent isothermal crystallization was studied by monitoring the modulus of the complex viscosity, $\eta^{*}$, during the crystallization process as a function of time, t. Figure 3A shows the effect of crystallization temperature on the evolution of the modulus of the complex viscosity for PA1. At short times, the material is in its supercooled state but viscosity is still low. However, at a certain time, viscosity shoots up which is indicative of crystallization. From these curves, a characteristic time-scale, $t_{c h a r, q}$, can be determined, that can serve as a comparative time-scale between different experiments. The choice of this time is somewhat arbitrary and here it is chosen as the inflection point of the viscosity curve as shown in Fig. 3A. As can be seen from this curve, lowering the crystallization temperature speeds up crystallization as expected in this temperature range (e.g. Acierno and Van Puyvelde, 2005).

Figure $3 \mathrm{~B}$ shows the modulus of the complex viscosity for the three molecular weights under study at a crystallization temperature of $202^{\circ} \mathrm{C}$. The starting values of the viscosity are of course different because of the different molecular weights. From this figure, it is clear that in the case of quiescent conditions, $t_{c h a r, q}$ is found to increase slightly with increasing molecular weight. This is again an expected result, given the fact that an increased molecular weight decreases chain mobility and hence will hamper crystallization. 


\subsection{Flow-Induced Crystallization}

This section reports on the flow-induced crystallization experiments using the short-term shearing protocol explained in Fig. 1. As was already mentioned in the Materials and Methods section, the shearing time has been kept constant at $1 \mathrm{~s}$. To follow the crystallization process, the modulus of the complex viscosity was monitored, as in the case of quiescent crystallization. Also here, a characteristic time for crystallization was defined as the inflection point of the viscosity curve. An example of such an evolution is given in Fig. 4A in which a quiescent crystallization experiment is compared with a flow-induced crystallization experiment performed on PA2 in which a shear rate of $5 \mathrm{~s}^{-1}$ has been applied. As can be seen, the crystallization after a shear pulse of $5 \mathrm{~s}^{-1}$ clearly accelerates the crystallization kinetics, as was expected based on experiments performed on other polymers. The effect of different shear rates is demonstrated in Fig. 4B, in which it is shown that higher shear rates increase the crystallization kinetics even more. In order to compare the different polyamide grades and to test the scaling relations valid for polyolefins, a dimensionless crystallization time $\theta$ is defined as the characteristic time divided by the characteristic time of quiescent crystallization.

In order to determine the different flow-induced crystallization regimes, two different Weissenberg numbers are constructed. The first one, $\mathrm{Wi}_{\text {rep }}$, is defined as the shear rate $\dot{\gamma}$ multiplied with the longest relaxation time of the polymer as defined in the previous section. As in the case of polyolefines, if this value is smaller than 1 , flow is not fast enough to induce orientation in the entangled melt and no significant influence on the crystallization kinetics should be expected. Above 1, orientational effects should start to play a role and an acceleration of the crystallization kinetics is anticipated. A second Weissen- berg number, $\mathrm{Wi}_{\mathrm{s}}$ is based on the onset of chain stretching expressed by the product of the shear rate $\dot{\gamma}$ and the relaxation time $\tau_{\text {Rouse }}$ (Eq. 2). When this Weissenberg number is smaller than 1 , no chain stretching is expected and hence no formation of shish-kebab structures is anticipated. Above a value of 1 , shish-kebab structures are prone to appear.

The dimensionless characteristic crystallization time is plotted as a function of both Weissenberg numbers in Figs. 5A $\left(\mathrm{Wi}_{\text {rep }}\right)$ and $5 \mathrm{~B}\left(\mathrm{Wi}_{\mathrm{s}}\right)$. Regarding the effect of $\mathrm{Wi}_{\text {rep }}$, it is seen that for values below 1 , the dimensionless crystallization time is close to 1 , indicating that the imposed flow did not have a significant effect on the crystallization kinetics. However, when $\mathrm{Wi}_{\text {rep }}$ exceeds 1 , a significant acceleration of the crystallization process is observed. When examining Fig. 5B, it is seen that the values of $\mathrm{Wi}_{\mathrm{s}}$ are still far below a value of 1 . Hence it is not expected to observe any highly oriented shishkebab related structures yet in these samples. However, the effect of molecular weight is slightly better captured with $\mathrm{Wi}_{\mathrm{s}}$ as the curves are closer together than when using $\mathrm{Wi}_{\text {rep }}$. Despite of the quality of the scaling, it can be concluded that the same scalings used for polyolefins can be applied to these polyamides, at least to predict the onset of crystallization acceleration. However, in order to validate the scaling for the chain stretching and the formation of highly oriented structures at values of $\mathrm{Wi}_{\mathrm{s}}$ close or larger than 1 , it is clear that the shear rates reached in these experiments are insufficient. When the shear rates become too large, shear fracture occurs which hampers a good interpretation of the rheological signal and hence the modulus of the complex viscosity cannot be followed anymore as a function of time. In order to boost the shear rates closer to $\mathrm{Wi}_{\mathrm{s}}$ of 1 , another approach was followed.

In this approach, the rheometer is not used to collect rheological data but to prepare the polyamide sample for further ana-
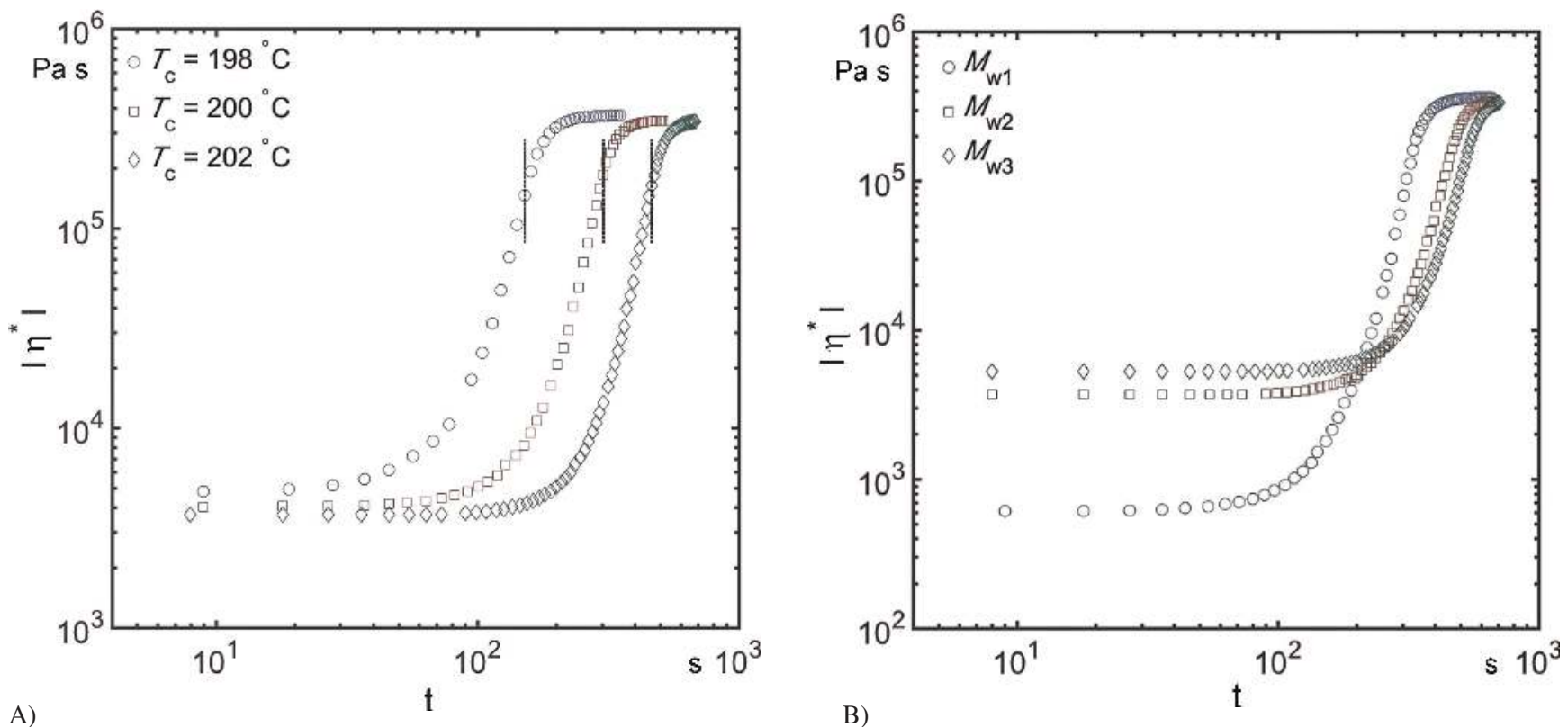

Fig. 3. Modulus of complex viscosity versus time at different crystallization temperatures for PA2 (A), modulus of complex viscosity versus time at different molecular weights at $T_{c}=202{ }^{\circ} \mathrm{C}(\mathrm{B})$. Both measurements are carried out at an angular frequency of $10 \mathrm{rad} / \mathrm{s}$

Intern. Polymer Processing XXXIII (2018) 3 
lysis. Hereto, the sample is sheared for $1 \mathrm{~s}$ at shear rates up to $250 \mathrm{~s}^{-1}$. The latter is a limiting value since above these shear rates, the rheometer goes into overload. This shear rate is - according to the Rouse relaxation times of these polymers - still insufficient to reach $\mathrm{Wi}_{\mathrm{s}}$ values well above 1 . However, values closer to 1 can be reached compared to the classical rheometer experiments so that at least trends towards more orientation could still be identified. Since the polyamides contain a small amount of mould release agents, the samples can easily be recuperated. In order to verify whether shish-kebab like structures are developing, WAXS experiments have been con- ducted. This approach has also be conducted for small values of the shear rate to verify the non-existing contribution of flow to the crystallization at $\mathrm{Wi}_{\text {rep }}<1$.

An example of WAXS-data is shown in Fig. 6. As can be seen, at small values of the shear rate and hence small values of the Deborah numbers, the WAXS patterns are isotropic, hence pointing towards an absence of the influence of flow on orientation although flow can still enhance point nucleation. When $\mathrm{Wi}_{\text {rep }}$ becomes larger than 1 , some orientation becomes visible since the isotropic character of the pattern is lost. At larger values of the shear rate, a clear Bragg peak at zero azi-
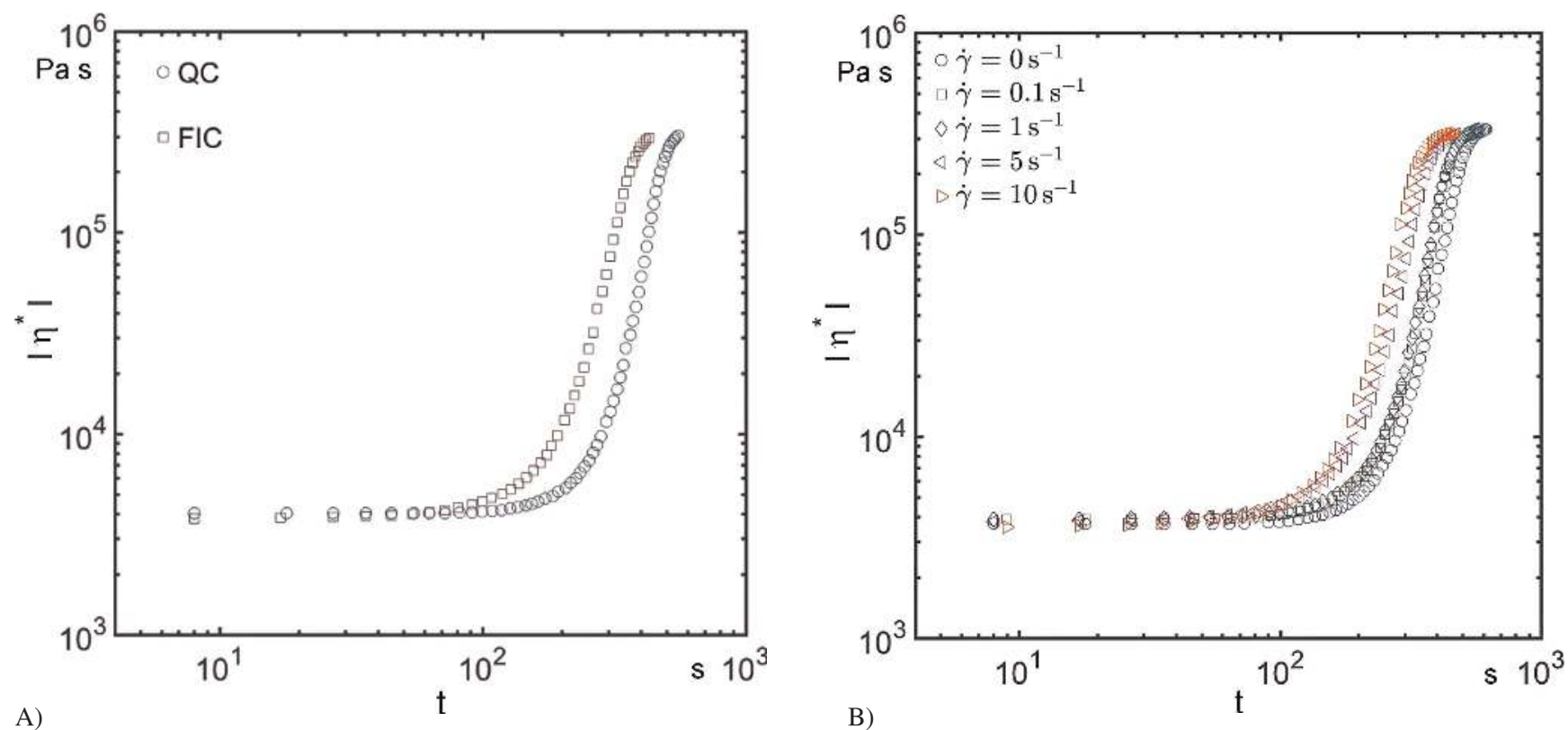

Fig. 4. Modulus of complex viscosity versus time for PA2 at $T_{c}=202^{\circ} \mathrm{C}, \dot{\gamma}=5 \mathrm{~s}^{-1}$ and $\omega=10 \mathrm{rad} / \mathrm{s}$, A) quiescent and under flow crystallization, $B)$ shear-induced crystallization at various shear rates
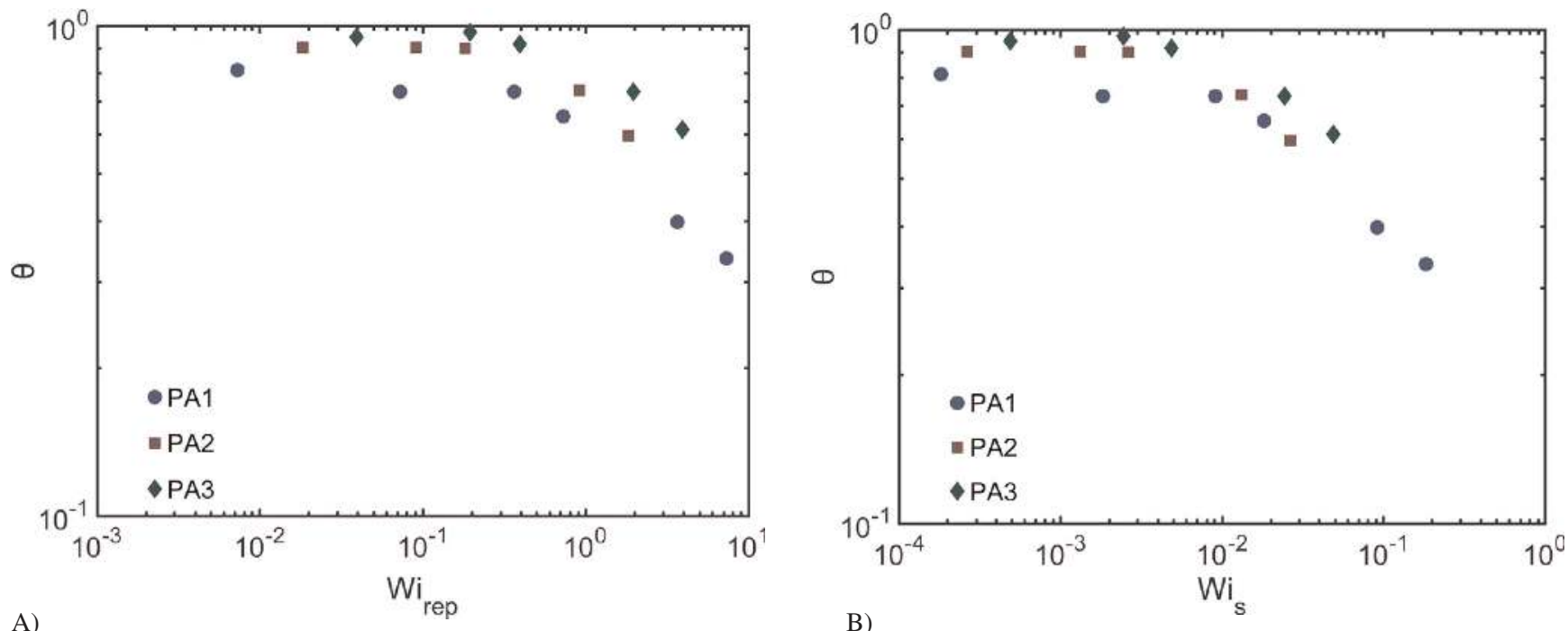

A)

Fig. 5. Evolution of the dimensionless crystallization time, $\theta$, for the different molecular weight samples at $T_{c}=202^{\circ} \mathrm{C}$ as a function of A) Wi $i_{\text {rep }}$ and $B) W i_{s}$ 

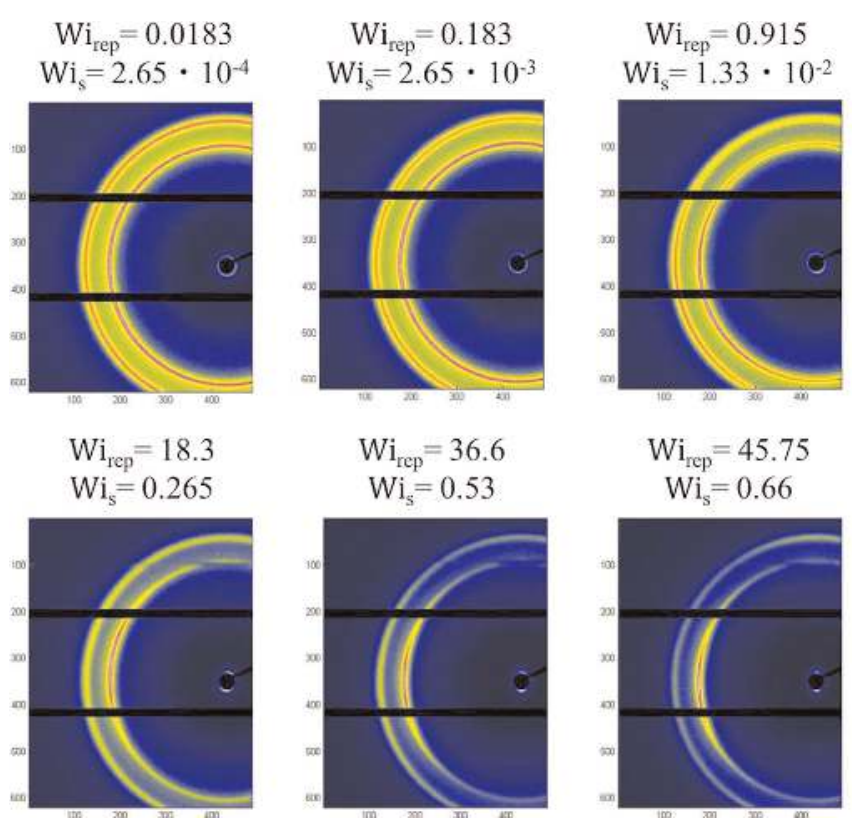

muthal angle is developing which points towards the development of significantly oriented structures.

Figure 7 shows the intensity as a function of scattering angle of the patterns obtained at different shear rates. As can be seen, the curves show two distinct peaks at angles $2 \theta=20^{\circ}$ and at $2 \theta=23^{\circ}$. This two peak nature indicates that the sample has crystallized in the $\alpha$-form and that this polymorph is independent whether flow has been applied or not (Chandra et al., 2005; Dasgupta et al., 1996; Yebra-Rodríguez et al., 2009). Usually, when no shear is applied, the $\gamma$-form should be present. However, when the polymer has seen an extrusion step as is the case for our polyamides since they have been compounded with mould release agent - this $\gamma$-form is transferred to the $\alpha$-form as also indicated by Khanna et al. (1988). This memory effect cannot be canceled anymore by using high annealing temperatures as is also seen in our results.

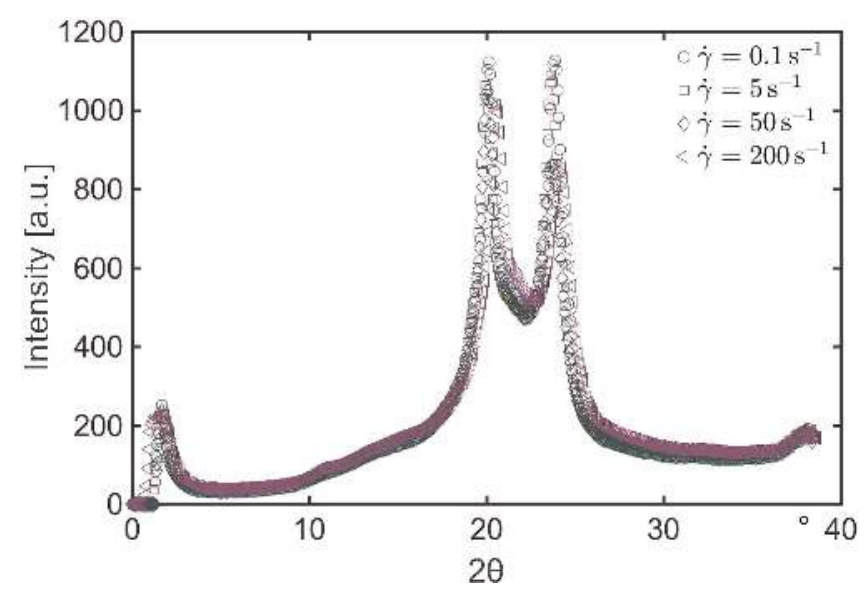

Fig. 7. X-ray diffraction pattern obtained during the isothermal crystallization of the grade PA2 at $202^{\circ} \mathrm{C}$

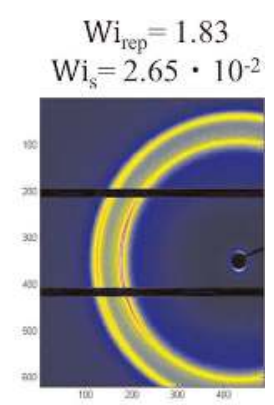

Fig. 6. $2 D$ diffraction pattern of the grade $P A 2$, pre-treated with different shear rates

To quantify the degree of orientation, the reflection of the Bragg peak closest to the center of the pattern was treated by the 'Full Width at half Maximum' method (FWHM) (Van Drongelen et al., 2014). This method fits a Lorentzian function to the azimuthal intensity profiles according to:

$\mathrm{I}_{\mathrm{L}}(\beta)=\mathrm{A}+\frac{\mathrm{B} \cdot \gamma}{\left(\beta-\beta_{0}\right)^{2}+\gamma^{2}}$,

where $\mathrm{A}$ and $\mathrm{B}$ are scaling parameters, $\beta_{0}$ the center of a peak and $\gamma$ a parameter specifying the width. The FWHM is then given as $2 \gamma$. A smaller value of FWHM indicates a sharper Bragg peak and hence is a quantitative measure of the overall orientation.

An example of this approach is given in Fig. 8. Here the inverse of FWHM is plotted as a function of $\mathrm{Wi}_{\mathrm{s}}$ for PA2. As can be seen, the higher the value of $\mathrm{Wi}_{\mathrm{s}}$, the smaller the peak width becomes as expressed by an increasing 1/FWHM-curve. This indeed indicates that orientation is clearly developing. As was already explained, full stretching is not yet achieved since the shear rates used in these experiments were not sufficient to reach $\mathrm{Wi}_{\mathrm{s}}$ numbers well above 1 . This is also clear from Fig. 8 since full orientation would lead to a plateau in the curve. However, from Fig. 8, it is clear that orientation is becoming more and more dominant as $\mathrm{Wi}_{\mathrm{s}}$ increases. Hence, the simple scaling relations based on $\mathrm{Wi}_{\text {rep }}$ and $\mathrm{Wi}_{\mathrm{s}}$ provide a good means to estimate the effect of shear rates on the flow-induced crystallization experiments.

\section{Conclusions}

This paper discussed the effect of shear on the crystallization of different polyamide- 6 samples. The rheological characterization of the samples yielded the longest relaxation time and the Rouse relaxation time. These times were used to estimate the transition Weissenberg numbers to indicate what the effect of 


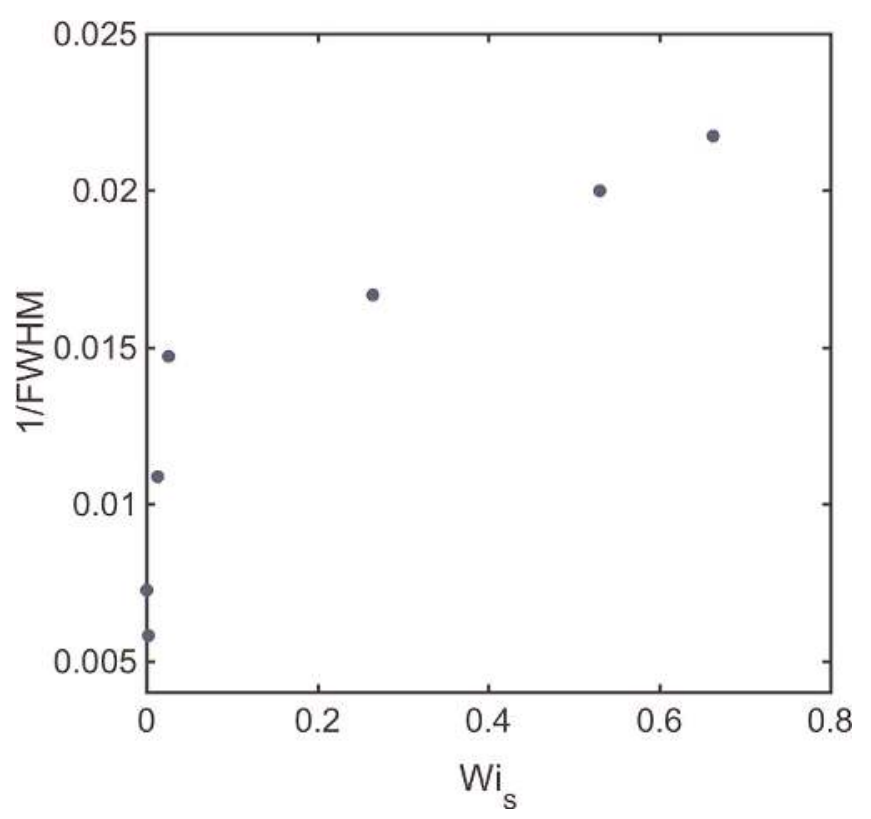

Fig. 8. 1/FWHM versus $W i_{s}$ for the grade PA2

shear rate would be on the crystallization process. These concepts were already tested to a large extent on polyolefin-like materials but not yet on other polymer families like for instance polyamide- 6 . The results have demonstrated that $\mathrm{Wi}_{\text {rep }}$ can be used to determine whether an acceleration of the crystallization kinetics - induced by flow - can be anticipated. On the other hand, the use of $\mathrm{Wi}_{\mathrm{s}}$ can be used to determine whether a significant degree of orientation can be expected in the sample. The results indicate that the simple scaling relations that were originally determined for polyolefins can be extended to this polyamide-6 family.

\section{References}

Acierno, S., Palomba, B., Winter, H. H. and Grizzuti, N., "Effect of Molecular Weight on the Flow-Induced Crystallization of Isotactic Poly(1-butene)", Rheol. Acta, 42, 243-250 (2003), DOI: $10.1007 / \mathrm{s} 00397-002-0280-9$

Acierno, S., Van Puyvelde, P., "Effect of Short Chain Branching upon the Crystallization of Model Polyamides-11", Polymer, 46, 10331 - 10338 (2005), DOI:10.1016/j.polymer.2005.07.097

Acierno, S., Grizzuti, N., "Flow-Induced Crystallization of Polymer: Theory and Experiments", Int. J. Mater. Form., 1, 583-586 (2008), DOI:10.1007/s12289-008-0323-6

Acierno, S., Coppola, S. and Grizzuti, N., "Effects of Molecular Weight Distribution on the Flow-Enhanced Crystallization of Poly(1-butene)", J. Rheol., 52, 551-566 (2008), DOI: $10.1122 / 1.2829149$

Baert, J., Van Puyvelde, P., "Effect of Molecular And Processing Parameters On The Flow-Induced Crystallization of Poly-1-Butene. Part 1: Kinetics and Morphology", Polymer, 47, 5871-5879 (2006), DOI:10.1016/j.polymer.2006.06.009

Baert J., Van Puyvelde, P. and Langouche, F., "Flow-Induced Crystallization of PB-1: From the Low Shear Rate Region up to Processing Rates", Macromolecules, 39, 9215-9222 (2006), DOI:10.1021/ma062068q
Chandra, A., Gong, S., Yuan, M., Turng, L. S., Gramann, P. and Cordes, H., "Microstructure and Crystallography in Microcellular Injection-Molded Polyamide-6 Nanocomposite and Neat Resin", Polym. Eng. Sci., 45, 52-61 (2005), DOI:10.1002/pen.20229

Dasgupta, S., Hammond, W. B. and Goddard III, W. A., "Crystal Structures and Properties of Nylon Polymers from Theory", J. Am. Chem. Soc., 118, 12291 - 12301 (1996), DOI:10.1021/ja944125d

Ebewele, R. O.: Polymer Science and Technology, CRC Press LLC, Boca Raton (2000), DOI:10.1201/9781420057805

Ferry, J. D.: Viscoelastic Properties of Polymers, 3rd Edition, Wiley, New York (1980)

Gao, Y., Dong, X., Wang, L., Liu, G., Liu, X., Tuinea-Bobe, C., Whiteside, B., Coates, P., Wang, D. and Han, C. C., "Flow-Induced Crystallization of Long Chain Aliphatic Polyamides under a Complex Flow Field: Inverted Anisotropic Structure and Formation Mechanism", Polymer, 73, $91-101$ (2015), DOI:10.1016/j.polymer.2015.07.029

Gowariker, V. R., Viswanathan, N. V. and Sreedhar, J.: Polymer Science, Halsted Press (John Wiley \& Sons), New York (1986)

Hamad, F. G., Colby, R. H. and Milner, S. T., "Lifetime of Flow-Induced Precursors in Isotactic Polypropylene", Macromolecules, 48, 7286-7299 (2015), DOI:10.1021/acs.macromol.5b00386

Harper, C. A., Petrie, E. M.: Plastics Materials and Processes: A Concise Encyclopedia, Wiley- Interscience, New York (2003), DOI: $10.1002 / 0471459216$

Housmans, J. W., Peters, G. W. M. and Meijer, H. E. H., "Flow-Induced Crystallization of Propylene/Ethylene Random Copolymers", J. Therm. Anal. Calorim., 98, 693-705 (2009), DOI: $10.1007 / \mathrm{s} 10973-009-0532-3$

Housmans, J. W., Steenbakkers, R. J. A., Roozemond, P. C., Peters, G. W. M. and Meijer, H. E. H., "Saturation of Pointlike Nuclei and the Transition to Oriented Structures in Flow-Induced Crystallization of Isotactic Polypropylene", Macromolecules, 42, 5728 5740 (2009), DOI:10.1021/ma802479c

Kelnar, I., Kratochvíl, J. and Mikešová, J., "Shear Flow Effect on the Crystalline Forms in Polyamide 6/Montmorillonite Nanocomposites", J. Appl. Polym. Sci., 106, 3387-3393 (2007), DOI:10.1002/app.27021

Khanna, Y. P., Kumar, R. and Reimschuessel, A. C., "Memory Effects in Polymers. I. Orientational Memory in the Molten State; its Relationship to Polymer Structure and Influence on Recrystallization Rate and Morphology", J. Appl. Polym. Sci., 35, 2259-2268 (1988), DOI:10.1002/app.1988.070350824

Khanna, Y. P., Han, P. K. and Day, E. D., "New Developments in the Melt Rheology of Nylons. I: Effect of Moisture and Molecular Weight", Polym. Eng. Sci., 36, 1745-1754 (1996), DOI: $10.1002 /$ pen. 10570

Kohan, M. I.: Nylon Plastics, John Wiley \& Sons, New York (1973)

Kumaraswamy, G., Issaian, A. M. and Kornfield, J. A., "Shear-Enhanced Crystallization in Isotactic Polypropylene. 1. Correspondence between in situ Rheo-Optics and ex situ Structure Determination", Macromolecules, 32, 7537-7547 (1999), DOI: $10.1021 / \mathrm{ma} 990772 \mathrm{j}$

Kumaraswamy, G., Verma, R. K., Issaian, A. M., Wang, P., Kornfield, J. A., Yeh, F., Hsiao, B. S. and Olley, R. H. "Shear-Enhanced Crystallization in Isotactic Polypropylene. 2. Analysis of the Formation of the Oriented Skin", Polymer, 41, 8931-8940 (2000), DOI: 10.1016/S0032-3861(00)00236-6

Lamberti, G., "Flow Induced Crystallization of Polymers", The Royal Society of Chemistry, 43, 2240-2252 (2014), PMid:24202547; DOI: $10.1039 / \mathrm{c} 3 \mathrm{cs} 60308 \mathrm{c}$

Liedauer, S., Eder, G., Janeschitz-Kriegl, H., Jerschow, P., Geymayer, W. and Ingolic, E., "On the Kinetics of Shear Induced Crystallization in Polypropylene", Int. Polym. Proc., 8, 236-244 (1993), DOI: $10.3139 / 217.930236$

Ma, Z., Balzano, L., Van Erp, T., Portale, G., and Peters, G. W. M., "Short-Term Flow Induced Crystallization in Polypropylene: How Short is Short?", Macromolecules, 46 9249-9258 (2013), DOI: $10.1021 / \mathrm{ma} 401833 \mathrm{k}$

Mark, H., Stafford Whitby, G.: Collected Papers of Wallace Hume Carothers on High Polymeric Substances, High Polymers, Interscience, New York (1940), PMid:17857663 
Naudy, S., David, L., Rochas, C. and Fulchiron, R., "Shear Induced Crystallization of Poly(m-xylylene adipamide) with and without Nucleating Additives", Polymer, 48, 3273-3285 (2007), DOI: 10.1016/j.polymer.2007.03.076

Naudy, S., Fulchiron, R., "Static and Shear Induced Crystallization of Glass Fiber Reinforced Poly(m-xylylene adipamide) with Nucleating Additives", J. Polym. Sci., Part B: Polym. Phys., 45, 29822992 (2007), DOI: 10.1002/polb.21288

Seki, M., Thurman, D. W., Oberhauser, J. P.and Kornfield, J. A., "Shear-Mediated Crystallization of Isotactic Polypropylene: The Role of Long Chain-Long Chain Overlap", Macromolecules, 35, 2583 -2594 (2002), DOI:10.1021/ma011359q

Somani, R. H., Hsiao, B. S., Nogales, A., Srinivas, S., Tsou, A. H., Sics, I., Balta- Calleja, F. J. and Ezquerra, T. A., "Structure Development during Shear Flow Induced Crystallization of I-PP: In-situ Small Angle X-Ray Scattering Study", Macromolecules, 33, 9385-9394 (2000), DOI:10.1021/ma001124z

Tavernier, B., Mewis, J., Van Puyvelde, P., Takenaka, M., Ernst, B. and Hashimoto, T." "Effect of Thermomechanical History on the Crystallization of Poly(ether-block-amide)", Polym. Eng. Sci., 48, 2418-2425 (2008), DOI:10.1002/pen.21197

Van Drongelen, M., Cavallo, D., Balzano, L., Portale, G., Vittorias, I., Bras, W., Alfonso, G. C. and Peters, G. W. M., "Structure Development of Low-Density Polyethylenes during Film Blowing: A RealTime Wide-Angle X-ray Diffraction Study", Macromolecular Journals, 299, 1494-1512 (2014), DOI:10.1002/mame.201400161

Van Meerveld, J., Peters, G. W. M. and Hutter, M., "Towards a Rheological Classification of Flow Induced Crystallization Experiments of Polymer Melts", Rheol. Acta, 44, 119-34 (2004), DOI:10.1007/s00397-004-0382-7

\section{Appendix A}

\begin{tabular}{|c|c|c|c|}
\hline $\begin{array}{c}\mathrm{T} \\
{ }^{\circ} \mathrm{C}\end{array}$ & $\alpha_{\mathrm{T}}$ & $\begin{array}{c}\tau_{\text {rep }} \\
\mathrm{s}\end{array}$ & $\begin{array}{c}\tau_{\text {Rouse }} \\
\mathrm{s}\end{array}$ \\
\hline 240 & 1 & 0.04 & $9.98 \cdot 10^{-4}$ \\
198 & 2.045 & 0.082 & $2.04 \cdot 10^{-3}$ \\
200 & 1.934 & 0.077 & $1.93 \cdot 10^{-3}$ \\
202 & 1.830 & 0.073 & $1.83 \cdot 10^{-3}$ \\
\hline
\end{tabular}

Table A.1. Rheological time-scales at $T=202^{\circ} \mathrm{C}$ for PA1

\begin{tabular}{|c|c|c|c|}
\hline $\begin{array}{c}\mathrm{T} \\
{ }^{\circ} \mathrm{C}\end{array}$ & $\alpha_{\mathrm{T}}$ & $\begin{array}{c}\tau_{\text {rep }} \\
\mathrm{s}\end{array}$ & $\begin{array}{c}\tau_{\text {Rouse }} \\
\mathrm{s}\end{array}$ \\
\hline 240 & 1 & 0.1 & $1.44 \cdot 10^{-4}$ \\
198 & 2.048 & 0.205 & $2.96 \cdot 10^{-3}$ \\
200 & 1.937 & 0.194 & $2.8 \cdot 10^{-3}$ \\
202 & 1.833 & 0.183 & $2.65 \cdot 10^{--3}$ \\
\hline
\end{tabular}

Table A.2. Rheological time-scales at $T=202^{\circ} \mathrm{C}$ for $P A 2$
Vega, J. F., Hristova, D. G. and Peters, G. W. M., "Flow-Induce Crystallization Regimes and Rheology of Isotactic Polypropylene: Effects of Molecular Architecture", J. Therm. Anal. Calorim., 98, 655-666 (2009), DOI:10.1007/s10973-009-0516-3

Vleeshouwers, S., Meijer, H. E. H., "A Rheological Study of Shear Induced Crystallization", Rheol. Acta, 35, 391-399 (1996), DOI: $10.1007 /$ BF00368990

Yebra-Rodríguez, A., Alvarez-Lloret, P., Cardell, C. and RodríguezNavarro, A. B., "Crystalline Properties of Injection Molded Polyamide-6 and Polyamide-6/Montmorillonite Nanocomposites", Appl. Clay Sci., 43, $91-97$ (2009),

DOI:10.1016/j.clay.2008.07.010

Date received: June 15, 2017

Date accepted: August 04, 2017

Bibliography

DOl 10.3139/217.3524

Intern. Polymer Processing

XXXIII (2018) 3; page 327-335

(c) Carl Hanser Verlag $\mathrm{GmbH} \&$ Co. KG

ISSN 0930-777X

\begin{tabular}{|c|c|c|c|}
\hline $\begin{array}{c}\mathrm{T} \\
{ }^{\circ} \mathrm{C}\end{array}$ & $\alpha_{\mathrm{T}}$ & $\begin{array}{c}\tau_{\text {rep }} \\
\mathrm{s}\end{array}$ & $\begin{array}{c}\tau_{\text {Rouse }} \\
\mathrm{s}\end{array}$ \\
\hline 240 & 1 & 0.14 & $1.75 \cdot 10^{-3}$ \\
198 & 3.160 & 0.442 & $5.52 \cdot 10^{-3}$ \\
200 & 2.978 & 0.417 & $5.20 \cdot 10^{-3}$ \\
202 & 2.807 & 0.393 & $4.90 \cdot 10^{-3}$ \\
\hline
\end{tabular}

Table A.3. Rheological time-scales at $T=202^{\circ} \mathrm{C}$ for $P A 3$ 